\title{
Giant Cardiac Fibroma: An Unusual Cause of Failure to Thrive
}

\author{
Susanne Navarini · Philipp Latzin • \\ Alexander Kadner · Thierry Carrel • \\ Damian Hutter
}

Received: 27 January 2012/Accepted: 8 May 2012/Published online: 30 May 2012

(C) Springer Science+Business Media, LLC 2012

\begin{abstract}
Cardiac fibromas are extremely rare in the general pediatric population and may present with a wide spectrum of clinical signs, including life-threatening arrhythmias and sudden death. We report a 14-month-old boy who presented with failure to thrive as the only symptom. Echocardiography showed a large cardiac fibroma in the right ventricle. Cardiac magnetic resonance imaging confirmed the diagnosis. After complete surgical tumor resection, the boy showed normal catch-up growth. This case underlines the diversity of clinical features of cardiac tumors, which implies that they should be considered early in the differential diagnosis of infants with failure to thrive.
\end{abstract}

Keywords Cardiac fibroma - Children - Failure to thrive . Infant

\section{Introduction}

Primary cardiac tumors are extremely rare in the pediatric population with a reported incidence of $0.027 \%$ at early

S. Navarini · D. Hutter $(\square)$

Division of Pediatric Cardiology, University Children's

Hospital of Berne, Berne, Switzerland

e-mail: damian.hutter@insel.ch

P. Latzin

Division of Pediatric Pulmonology, University Children's

Hospital of Berne, Berne, Switzerland

A. Kadner

Division of Pediatric Cardiac Surgery, Department of Cardiac

Surgery, University Hospital of Berne, Berne, Switzerland

T. Carrel

Division of Pediatric Cardiac surgery, University Hospital of Berne, Berne, Switzerland autopsy studies [9]. An observed increase in the incidence from $0.06 \%$ in the early 1980 s to $0.32 \%$ between 1990 and 1995 is presumably due to improvements in diagnostic imaging [2]. The majority of primary cardiac tumors are benign. The most common tumors in childhood are rhabdomyoma, followed by fibroma and teratoma [2]. These tumors may cause a variety of symptoms, depending on their size and location. They may lack in clinical symptoms or present with life-threatening cardiac events. Here we report a 14-month-old boy with a right-ventricular fibroma who presented with failure to thrive as the only clinical finding. After complete surgical resection of the tumor, the patient regained normal growth and normal weight for age.

\section{Case Report}

A 14-month-old, otherwise healthy boy presented to our cardiology outpatient clinic with failure to thrive to rule out a cardiac etiology. At the age of 4 months, he started to deviate from the 97 th percentile down to the 25 th percentile $(10 \mathrm{~kg})$ at the time of presentation (14 months). His length was $80 \mathrm{~cm}$ (50th percentile) (Fig. 1). The physical examination was normal except for a $2 / 6$ systolic ejection murmur, which was best audible along the left upper sternal border without radiation. The surface electrocardiogram showed a regular sinus rhythm with a heart rate of $112 / \mathrm{min}$, QRS axis $+60^{\circ}$, and incomplete right bundle branch block without ventricular hypertrophy. Despite a chest X-ray showing normal heart size and normal pulmonary vascular markings, and despite the lack of clinical signs for heart failure, a routine two-dimensional echocardiography was performed, which surprisingly showed a large intracardiac mass $(39 \times 23 \times 20 \mathrm{~mm})$ almost completely obstructing the right-ventricular cavity. There was 


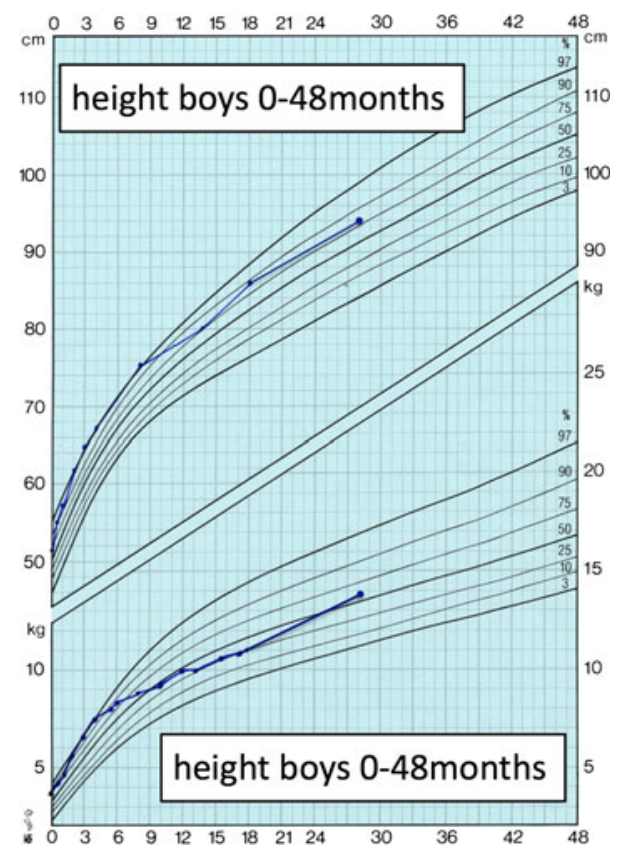

Fig. 1 Length-for-age and weight-for-age percentiles

mild systolic narrowing of the right-ventricular outflow tract (RVOT) but normal movement of the pulmonary valve as well as the tricuspid valve. Doppler examination showed turbulent flow during systole with mildly increased pressure gradient across the RVOT $(2.0 \mathrm{~m} / \mathrm{s})$. On cardiac magnetic resonance imaging (cMRI), tumor infiltration into the myocardial tissue of the free right-ventricular wall could not be excluded (Fig. 2). The patient was subsequently followed-up in our outpatient clinic with the tumor mass neither showing progression nor regression in size during a period of 12 weeks. During this time, the boy did not gain weight despite high-caloric tube feeding. He continued to have regular bowel movements. This led to the conclusion that the tumor had an important hemodynamic impact that was causing significant RVOT

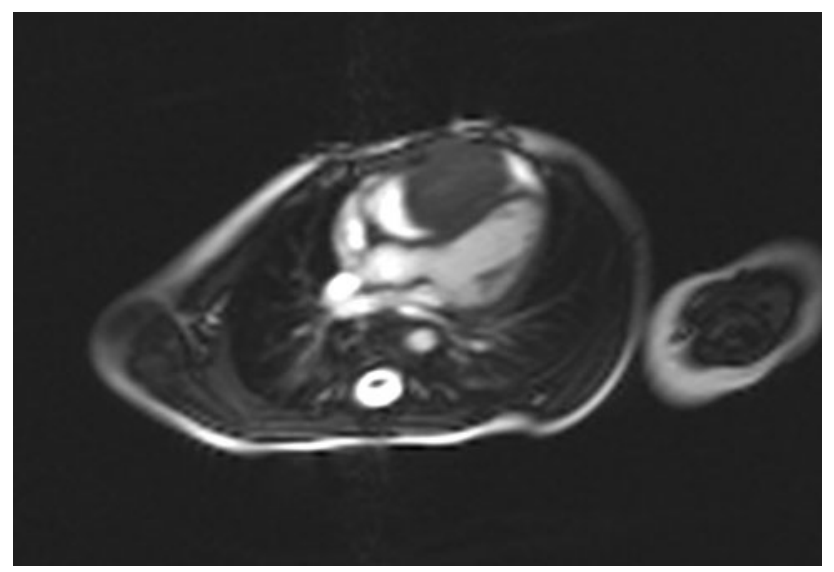

Fig. 2 cMRI showing a large intracardiac mass $(39 \times 23 \times 20 \mathrm{~mm})$ obstructing almost completely the right-ventricular cavity

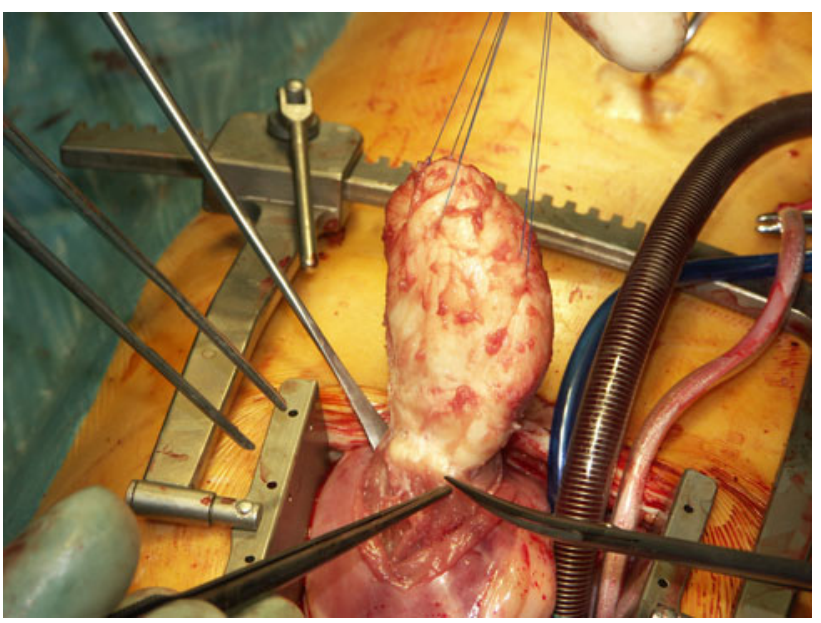

Fig. 3 Intraoperative finding of a grey-white solitary hard mass within the right-ventricular cavity

obstruction during systole. It was speculated that either intermittent decreased cardiac output due to ventricularventricular interaction or increased right atrial pressure leading to increased venous pressures might be responsible for the boy's failure to thrive. It was finally decided to surgically remove the tumor.

During surgery, a grey-white solitary hard mass was found. The mass almost completely obstructed the rightventricular cavity, only sparing the tricuspid valve, the RVOT, and the pulmonary valve. By way of a longitudinal right ventriculotomy, the tumor was removed in toto, and reconstruction of the right anterior wall with a GORE-TEX patch was performed. The mass weighed $17.5 \mathrm{~g}$ and measured $50 \times 30 \times 25 \mathrm{~mm}$ (Fig. 3). Histologic examination confirmed the intraoperative finding of a fibroma that was surrounded by a thin capsule and no central calcification or necrosis. The postsurgical course was uneventful. The patient was discharged from the pediatric intensive care unit to the ward 4 days after surgery. On day 8 after cardiovascular surgery, he was discharged from the hospital in excellent physical condition.

Fourteen months after surgery, the infant showed a normal gain in weight [13.9 $\mathrm{kg}$ (50th to 75 th percentile)] and length [94 cm (75th percentile)]. Echocardiographic findings showed normalized biventricular function with only mild regional wall motion abnormalities along the free right-ventricular wall. Electrocardiogram showed regular sinus rhythm with a heart beat of $135 / \mathrm{bpm}$, normal QRS axis, and complete right bundle branch block.

\section{Discussion}

Cardiac tumors are extremely rare findings in children with predominant benign histology $(\leq 90 \%)$ [1]. The most common cardiac tumors in childhood are rhabdomyomas 
with multiple lesions in both chambers. Over time they show partial or complete spontaneous regression in size in $\leq 50 \%$ of patients [2]. In contrast, cardiac fibromas are single, solid tumors and are usually located along the free left-ventricular wall or along the interventricular septum. Often they are diagnosed in children younger than 1 year old. They lack tendency for spontaneous regression. Histologically they belong to the group of connective tissue tumors and derive from fibroblasts. In $\sim 25 \%$ of these tumors, central calcification is present, helping to distinguish the tumor from a single rhabdomyoma [3].

The clinical manifestation of a fibroma mainly depends on its size and intracardiac location. A large mass within the right-ventricular cavity, such as the one described in our patient, may limit diastolic filling of the right ventricle as well as emptying it during systole, thus leading to clinical signs of right heart failure. The mass obstruction of the tricuspid valve inflow behaves like a valvular tricuspid stenosis, whereas obstruction of the RVOT rather mimics a functional pulmonary stenosis. Both clinically manifest with symptoms of respiratory failure and/or right heart failure. Left-sided tumors show similar mechanisms [6]. In addition to the more obvious mass component effect with hemodynamic important compromise of ventricular filling and emptying, life-threatening arrhythmias are observed in up to 10 to $15 \%$ of patients. The tumor either incorporates conduction tissue, or its surface acts as an "artificial" accessory pathway. In some cases, compression of the native conduction system by the tumor may lead to various block manifestations [10]. The most commonly observed arrhythmias are ventricular tachycardia and fibrillation [8]. Complete atrioventricular and bundle branch blocks are less frequent. Rarely do patients present with supraventricular arrhythmias.

In our patient, neither right heart failure nor respiratory or gastrointestinal symptoms were present, which made us speculate that the hemodynamic impact of the tumor mass was not persistent but enough to significantly modulate cardiac output or central venous pressures.

Surgical intervention depends on type, size, and location of the tumor. Urgent primary resection is indicated whenever the patient experiences life-threatening symptoms either due to arrhythmia or due to hemodynamic important obstruction of inflow or outflow tracts. Given the high potential for lethal arrhythmias, some investigators favour primary surgical resection even in asymptomatic patients $[4,5]$. If complete tumor resection is not possible, partial resection may be beneficial. Palliation with cavopulmonary anastomosis or heart transplantation have been reported [7].

\section{Conclusion}

We report a 14-months-old boy with failure to thrive due to a giant right-ventricular intracardiac fibroma. Diagnosis was made with two-dimensional echocardiography and confirmed by cMRI. After surgical tumor resection, the infant regained adequate catch-up-growth.

Although cardiac fibromas may present with a wide spectrum of clinical features, this is the first report of an infant with failure to thrive as the only clinical finding. Primary cardiac tumors must be included in the differential diagnosis of syncope, arrhythmia, respiratory symptoms, seizures, and cyanosis in childhood. Given the clinical course of our patient, we therefore strongly suggest that unexplained failure to thrive in infancy should alert pediatricians for early consideration of intracardiac tumors in their differential diagnosis.

\section{References}

1. Abushaban L, Denham B, Duff D (1993) 10 year review of cardiac tumours in childhood. Br Heart J 70(2):166-169

2. Beghetti M, Gow RM, Haney I, Mawson J, Williams WG, Freedom RM (1977) Pediatric primary benign cardiac tumors: a 15-year review. Am Heart J 134(6):1107-1114

3. Burke AP, de Rosado Christenson M, Templeton PA, Virmani R (1994) Cardiac fibroma: clinicopathologic correlates and surgical treatment. J Thorac Cardiovasc Surg 108(5):862-870

4. Busch U, Kampmann C, Meyer R, Sandring KH, Hausdorf G, Konertz W (1995) Removal of a giant cardiac fibroma from a 4-year-old child. Tex Heart Inst J 22(3):261-264

5. Cho JM, Danielson GK, Puga FJ et al (2003) Surgical resection of ventricular cardiac fibromas: early and late results. Ann Thorac Surg 76(6):1929-1934

6. Gazit AZ, Gandhi SK (2007) Pediatric primary cardiac tumors: diagnosis and treatment. Curr Treat Options Cardiovasc Med 9(5):399-406

7. Gunther T, Schreiber C, Noebauer C, Eicken A, Lange R (2008) Treatment strategies for pediatric patients with primary cardiac and pericardial tumors: a 30-year review. Pediatr Cardiol 29(6): 1071-1076

8. Kusano KF, Ohe T (2002) Cardiac tumors that cause arrhythmias. Card Electrophysiol Rev 6(1-2):174-177

9. Nadas AS, Ellison RC (1968) Cardiac tumors in infancy. Am J Cardiol 21(3):236-363

10. Ottaviani G, Rossi L, Ramos SG, Matturri L (1999) Pathology of the heart and conduction system in a case of sudden death due to a cardiac fibroma in a 6-month-old child. Cardiovasc Pathol 8(2):109-112 